\title{
Pour une éducation relative à l'environnement réflexive
}

Lucie Sauvé et Louis Goffin

\section{(2) OpenEdition \\ Journals}

Édition électronique

URL : https://journals.openedition.org/ere/7118

DOI : $10.4000 /$ ere. 7118

ISSN : 2561-2271

Éditeur

Centr'ERE

Référence électronique

Lucie Sauvé et Louis Goffin, "Pour une éducation relative à l'environnement réflexive », Éducation relative à l'environnement [En ligne], Volume 1 | 1999, mis en ligne le 15 septembre 1999, consulté le 28 mai 2021. URL : http://journals.openedition.org/ere/7118; DOI : https://doi.org/10.4000/ere.7118 


\title{
Pour une éducation relative à l'environnement réflexive
}

\author{
Lucie Sauvé et Louis Goffin
}

1 L'éducation relative à l'environnement (ERE) évolue dans la mouvance des courants socioculturels actuels. Gardant un ancrage dans les premières propositions internationales de l'UNESCO au milieu des années 70, elle se transforme, évolue, s'enrichit, trouve des alliances fécondes; mais aussi parfois, elle risque l'atrophie, l'inflation ou autres formes de dérive. Quoi qu'il en soit, elle est de plus en plus prise en compte dans les discours éducationnels contemporains et elle devient partie intégrante de la plupart des nouveaux curriculums issus des réformes éducatives en cours. Après avoir été longtemps tributaire d'une sorte de «conspiration» d'éducateurs convaincus ${ }^{1}$, L'ERE entre dans une phase d'institutionnalisation.

2 Plus que jamais, la recherche est sollicitée comme support au développement d'une ERE qui soit crédible et utile. Par ailleurs, de plus en plus d'enseignants et d'animateurs prennent conscience de l'importance d'inscrire leur intervention éducative dans une démarche réflexive et critique, de façon à contribuer au développement de la recherche comme acteurs à part entière. Aux « $\mathrm{R}$ » désormais classiques du domaine de la gestion environnementale (Réduction, Récupération, Réutilisation-Recyclage) et aux « $\mathrm{R}$ » liés à la transformation des rapports humains au regard de l'environnement de la sphère de l'altérité (Responsabilité, Reconstruction des liens, Réorientation de nos choix sociaux), doivent s'ajouter ceux de la réflexivité : Regards, Recherches, Réflexions. Mais bien au-delà d'un ajout, il s'agit de promouvoir l'intégration des préoccupations d'intervention, d'éducation et de recherche, dans une perspective de synergie.

3 Cette nouvelle revue dont nous avons le très grand plaisir de signer le premier éditorial, a pour but de stimuler le développement de la recherche en ERE, de favoriser la diffusion et la discussion des activités en ce domaine au sein de la francophonie. Si la communauté internationale bénéficie déjà de plusieurs revues spécialisées en langue anglaise ${ }^{2}$, et si une revue hispanophone voit le jour cette année en Amérique latine ${ }^{3}$, la communauté des chercheurs francophones ne disposait jusqu'ici d'aucun créneau spécifique pour la diffusion de la discussion de ses recherches. Or, puisque le rapport à 
l'environnement est éminemment culturel, il importe de pouvoir publier dans sa propre langue et de faire valoir la richesse de cette dernière. Cela s'impose d'autant plus que dans le contexte mondialisation-globalisation actuel, la diversité culturelle fait face aux mêmes risques que la diversité biologique à laquelle elle est intimement liée.

Davantage encore que la diffusion de textes offrant des Regards, Recherches et Réflexions en ERE, la production de cette revue vise la mise en place d'un processus de coformation, d'échanges et de discussions critiques au sein de la communauté des chercheurs en ERE. L'enjeu n'est pas uniquement scientifique, il est également stratégique: il s'agit de stimuler le développement de l'ERE et de soutenir et de valoriser à cet effet les travaux des chercheurs, dont la plupart inscrivent leurs projets au cœur d'une pratique d'éducation ou de formation bien concrète.

5 L'idée-projet d'une telle revue est issue du Forum francophone international Planèt'ERE qui a réuni à Montréal, en novembre 1997, plus de 700 acteurs de l'ERE au sein de la francophonie. Plus précisément, le projet a émergé de discussions en table-ronde entre certains participants de Colloque international sur la recherche en éducation relative à l'environnement, organisé par l'Université du Québec à Montréal, dans le cadre du Forum. L'accord interinstitutionnel signé à cette même époque entre la Fondation Universitaire Luxembourgeoise (FUL, Belgique) et l'Institut des sciences de l'environnement de L'UQAM (Québec - Canada) a permis de consolider le projet. Deux autres partenaires se sont joints au comité éditorial : l'Ifrée (Institut de formation et de recherche en éducation à l'environnement, Poitou-Charentes, France) et l'Institut du Sahel (Mali).

6 À tour de rôle, chacune des institutions participantes se rendra responsable de l'édition $\mathrm{du}$ volume annuel, développant un thème particulier. Ainsi, ce premier volume, dont l'UQAM est responsable, aborde le thème de la recherche à partir des travaux présentés lors du Colloque tenu à Montréal. Le Volume 2, sous la responsabilité de la FUL, diffusera les travaux du Réseau REVERE - Réseau européen de recherche sur l'évaluation en éducation à l'environnement. L'Ifrée, dont l'une des préoccupations majeures est la formation des enseignants, préparera le Volume 3. Le volume 4 sera produit par l'Institut du Sahel et mettra en lien les enjeux de l'ERE avec ceux de l'éducation au développement.

7 Le thème de ce Volume 1, soit celui de la recherche elle-même, posait un défi particulier. Ce thème est ardu, faut-il le souligner. La communauté des chercheurs francophones en ERE commence à peine à se structurer et à développer sa propre culture de recherche. Étions-nous véritablement prêts à jeter un regard collectif, descriptif et critique, sur les caractéristiques et les enjeux de la recherche en ERE ? Ce volume témoigne de nos avancées actuelles, déjà importantes, mais il montre aussi de grands pans de nos travaux à développer et des avenues de co-formation à mettre en branle au sein de notre communauté de chercheurs. Et en ce sens, la publication de ce volume (processus et résultats) a atteint le but visé.

8 Mais n'y a-t-il pas à craindre que les propos de cet ouvrage ne rejoignent pas l'intérêt des praticiens qui sont davantage en quête de soutiens plus immédiats et concrets à leur action éducative ? Certes. L'un des défis que nous aurons à relever est celui de concilier le langage de la recherche - qui doit rester rigoureux, à la hauteur des standards scientifiques internationaux - et les exigences d'une communication auprès d'un public le plus large possible d'Acteurs en ERE, auprès desquels les activités de recherche doivent avoir des retombées, et chez lesquels il faut stimuler également le 
désir d'associer intervention, investigation et réflexion. Les praticiens doivent être conviés à devenir des acteurs privilégiés de la recherche en ERE.

À travers la section Recherches et Réflexions, qui présente les textes de conférences du Colloque tenu à Montréal, et à travers la section Regards, qui rapporte diverses préoccupations soulevées dans les communications et les tables-rondes de ce même Colloque, le lecteur trouvera diverses questions et analyses, abordera avec les auteurs différents objets et thèmes de recherche. La diversité des points de vue exprimés pourra sans doute faire l'objet de discussions et de débats. Nous souhaitons vivement que s'installe une dynamique interactive et critique autour des textes publiés dans les volumes successifs de la revue.

Outre les deux premières sections déjà mentionnées, la revue comporte également les sections suivants : Recension d'écrits récents qui présentent un regard, une réflexion ou une recherche en ERE; L'événement en ERE, comprenant des rapports critiques d'événements et des annonces d'événements à venir, en lien avec les « $\mathrm{R}$ » de la réflexivité ; Publications et ressources, dont le contenu contribue à nourrir la réflexion en ERE ou à diffuser des recherches; enfin, Répertoire de la recherche en ERE dont l'élaboration a été entreprise lors du Colloque tenu à Montréal, comme un processus de longue haleine visant à faire connaître et à valoriser la recherche en ERE.

11 En terminant, nous attirons l'Attention sur la page couverture de ce premier volume : on y retrouve une aquarelle de Frédéric Back, cet artiste québécois qui a toujours su allier l'engagement environnemental et la production artistique. Par ce choix éditorial, cette nouvelle revue de recherche en ERE veut contribuer à souligner les alliances fécondes entre Art et Environnement, et reconnaitre la dimension réflexive de l'activité artistique, qui nous invite à porter des regards neufs et parfois critiques sur les réalités de nos milieux de vie. Il peut s'agir aussi tout simplement d'une invitation à renouer avec la beauté des choses, comme ce Matin qui lève sa brume sur un paysage de la campagne québécoise. Chacun des prochains volumes nous offrira un regard artistique sur un paysage ou une réalité environnementale de l'un de nos pays ayant le français en partage.

12 Bonne lecture!

\section{NOTES}

1. "Conspirer, dans son sens littéral, signifie "souffler ensemble". Cela suppose à son principe, l'aspiration commune exercée par une espérance » (d'après Pierre Teilhard de Chardin, Dans Ferguson, M. (1981). Les enfants du Verseau - Pour un nouveau paradigme. Paris : Clamann-Lévy. P. 15).

2. Mentionnons les revues suivantes : The Journal of Environemental Education, Research in Environemental Education, Environmental Education and Communication, The Australian Journal of Environmental Education, The Canadian Journal of Environmental Education, The Journal of Geographical and Environmental Education Research.

3. Tôpicos en Educatión Ambiental. 


\section{AUTEURS}

\section{LUCIE SAUVÉ}

Université du Québec à Montréal

\section{LOUIS GOFFIN}

Fondation Universitaire Luxembourgeoise, Arlon, Belgique 